\title{
Robot Interface Design of domestic and condominium robot for ageing population
}

Niccolò Casiddu $^{1}$, Filippo Cavallo ${ }^{2}$, Alessandro Divano ${ }^{1}$, Irene Mannari ${ }^{2}$, Emanuele Micheli $^{1}$,Claudia Porfirione ${ }^{1}$, Matteo Zallio ${ }^{1}$, Michela Aquilano ${ }^{2}$, Paolo Dario ${ }^{2}$

\begin{abstract}
This paper presents a preliminary study to design a couple of robots, conceived to assist senior citizens $65+$ in domestic and public space. The design and development of these two robots, named Domestic and Condominium, concerned, from one hand, appropriate criteria of acceptability, usability, aesthetic and safety, and on the other hand specific functionalities to satisfy users' needs.
\end{abstract}

\section{Introduction}

European population projections have highlighted that the number of elderly people $65+$ in the world will quickly increase in the coming years [1]. Nowadays the society sustains elderly people to age well by means of medical cures, socio-medical services, and other social activities but these requests of support and assistance expected to become so high that it is becoming difficult to manage and sustain [2]. In addition the society is also facing the sustainability of the pension system that leads to an extension of the working life of senior citizens [3]. In this societal landscape, advanced robotic systems have the potential to drive tangible benefits in quality of life, improving citizens outcomes and reducing health and social costs.

"Robot Companions" is a concept that refers to a new generation of machines that will primarily help and assist citizens in daily activities at home, in their workplace and in other environments [4]. Robot Companions could be implemented as a plurality of complete advanced robotic services, integrated in intelligent environments, which will actively work in real conditions and cooperate with real people and between them to provide favourable independent living, improving the quality of life and the efficiency of care for elderly people. The innovation of this concept is to significantly enhance the performance and acceptability of the current ICT services for aging well to a new level of quality, provided by the cooperation of a plurality of robots and with the support of an Ambient Intelligence (AmI) infrastructure. Different robotic systems could be installed and integrated to cooperate and operate in domestic, condominium and outdoor environments (homes, hospitals, towns, public spaces), appropriately equipped with $\mathrm{AmI}$ infrastructure, and could implement an ecosystem that

\footnotetext{
${ }^{1}$ Dipartimento di Scienze per l'Architettura, Scuola Politecnica dell'Università di Genova, Italy, e-mail: casiddu@arch.unige.it

${ }^{2}$ The BioRobotics Institute, Scuola Superiore Sant'Anna, viale Rinaldo Piaggio 34, 56025 Pontedera (PI), Italy
} 
connects different stakeholders, such as elderly people and caregivers, social services, medical centres, municipalities, shops, pharmacies, etc.

This idea of "Robot Companions" is under investigation and development in the context of the Robot-Era Project (FP7-ICT-2011.7) that, sustained by a multidisciplinary teams from different technological, medical and industrial design disciplines, aims to design and implement social service robotics in a user and town centred design approach and characterized by a high level of technology and acceptability. Particularly the Robot-Era Project aims to develop, implement and demonstrate the general feasibility, scientific/technical effectiveness, social/legal plausibility and acceptability by end-users of advanced robotic services integrated with intelligent environments to provide help facilitate independent living, improving the quality of life and the efficiency of care for senior citizens.

This paper faces the design aspects of a couple of robots, conceived to assist senior citizens $65+$ in domestic and public space, and particularly focuses on the criteria of acceptability, usability, aesthetic and safety. The study highlights those properties that favour the human robot interaction, i.e. the affordance (immediately show its functionalities), safety (perceived safe), aesthetics (perceived familiar with an opportune combination of colours and soft / rigid materials), friendliness (emotionally accepted), usefulness (concretely useful in daily activities) and dependability (robust, effective and reliable).

\section{Robot Interface Design}

Interaction, Acceptability, Design process, Interface design, Usability.

Through the introduction of electronics and informatics the technological development, started in 1980s, had a profound impact on mass products, even in those fields

that were previously exclusive only to mechanics. Smartphones are an example of how the shapes of these modern devices are not bound to one univocal purpose or use, but this innovation in design can come at a cost. These devices are often difficult for "the non-alphabetized" in both their physical usability and their cognitive approach based on unusual and less intuitive behavioural codes. Hence, the "digital-divide" concept becomes a paradox, when taking into account the elderly population, that would potentially profit the most from a smart environment [15]. As a discipline, design has always been interested in physical interfaces based on specific ergonomic relations aimed to improve their use. During the mechanic era designing a product was a straightforward process: measurements were dictated by anthropometry, while functions determined the product shape. Unlike recent times, when mono-directional approaches were used solely in designing an interface, nowadays the AAL, the ICTs [10] and robotics in general are leading the way to new multidirectional scenarios: instead of focusing on machines themselves, research is showing more and more interest on 
the attention machines should foster towards human beings. In other words, this rapidly-advancing technological evolution leaves behind the dialogic interaction based on a dual pattern, in which the user acts and the calculator answers. Not to mention that thanks to these new scenarios, "friendly" and "usable" [11] products can be achieved and this means that they meet specific acceptance requirements such as safety, affordance (i.e. a sum of shape, dimension, weight, texture, ...), aesthetics and congeniality. Therefore it should be highlighted that interfaces cannot only be described as a means to connect human beings and products - interfaces are an open, dynamic and interactive system, able to ensure full accessibility to those advanced technologies, which effectively support an active ageing. Interface and object design also falls within the "project-making method": the most recent method is to move from centre to periphery or vice versa, by gradually approaching what will be recognized as the designed object, i.e. the final result. If designing means giving a shape, a function and a sense to ideas, then clearly there cannot be a design without a shape. In the field of Human Centred Robotics Design [9] a project is defined as a process on which designers, computer technicians and engineers work together as a team, sharing their planning expertise in order to develop a final product that is the result of all their synergetic inputs. Within this framework, the project aims to combine software and hardware components so that the device features may be controlled and programmed. Making a project today means facing this complexity and organizing it in an ordered system of different competences, and at the moment our part is a mere tile that will fall into place with other tiles to create a much wider mosaic.

\section{End-users' needs and service analysis}

The current design and development of the Robot-Era system is consequence of a substantial study, carried out with elderly people and caregivers with an User Centred Design (UCD) approach [5], that allowed to identify the primary end-user needs, possible structures of services to satisfy such needs and the scientific and technological requirements to develop the relative service robotic solutions. That study highlighted that a high willingness to use robotic systems in case of need was detected for transporting/manipulating objects at home, cleaning, garbage collection, surveillance, outdoor walking support, indoor escort at night and reminding events, laundry support, communicating with persons, for food delivery and shopping/drug delivery [6].

The main phases of the UCD methodology were carefully scheduled and included the (1) end-user needs analysis, (2) the design of services related to needs and the (3) production and implementation of robots for the testing phase. 


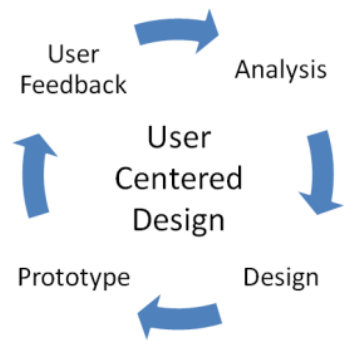

Figure 1 - Description of the UCD approach to design and develop the Robot-Era robotic services.

The first two phases were performed in three different European countries (Italy, Germany e Sweden), involving about 67 senior citizens 65+ and 17 caregivers. The study highlighted different end-users' needs that could be provided not only inside the domestic environment, but also outdoor in the public spaces. Table I describes the Robot-Era Services and the places where they are performed.

Table I - The Robot-Era services identified during the

\begin{tabular}{|l|l|l|}
\hline & Robot-Era Services & Environments \\
\hline 1) & Communication & Domestic environments \\
2) & Indoor escort at night & \\
$3)$ & Reminding & \\
$4)$ & Cleaning & \\
5) & Objects transportation and manipulation & Public spaces: squares, gardens, \\
\hline 6$)$ & Outdoor walking support & walking lanes, etc. \\
\hline 7$)$ & Laundry support & Condominium, Residential \\
8) & Food delivery & spaces, etc. \\
$9)$ & Surveillance & \\
\hline 10) & Drug and shopping delivery & Outdoor environments, shopping \\
11) & Garbage collection & centers, drug stores, etc. \\
\hline
\end{tabular}




\section{Shapes for Interaction}

Human Interaction, Machine Interaction, Safety.

People, machines, environments. They are the three main factors interacting within a complex ecosystem in constant evolution. People may be defined as "biological machines" gifted with thought, emotions and cultural heritage. In this context they communicate and interact with "new machines", which are now able to act and decide autonomously. Additionally, other types of interaction (besides people-machine interaction) take place within relatively confined environments, smart environments and in the urban ecosystem. The study on man-machine interaction has developed inside an experimental context involving the potential users, in order to improve the quality of autonomous life and the efficiency in the care of the elderly. [12] Based on a previous study, four fundamental acceptance parameters have been brought to light in man-machine relation:

- Safety: robot level of danger as perceived by the user.

- Aesthetics: robot capability to trigger familiar reactions in the user through its form, colours and materials used to build it.

- Recognisability: robot capability to highlight its function.

- Friendliness: robot capability to establish a positive emotional relation with the user.

Starting from the robot fundamental design characteristics, the present study focuses on volumes, shapes, the anthropometric relation, colours and material to be systemized for the development of the robot external shell. Moreover, this study analyses the main aspects regarding human perception of service robots with anthropomorphic features and the human acceptance of these features, which can be understood through shape symmetry, proportions and aspects specifically connected with those humans.

\subsection{Human Robot Interaction}

The new general asset of the robots discussed in this study is based on unity of shape derived from functions, coherence and recognisability of these shapes [13]. The aim of this design process is to boost the technical aspect of the robot family and its perform- 
ance, thus enabling it to provide a more efficient service. However, it should not be forgotten that final users are the main focus, together with their needs on various levels: ergonomic, functional, cognitive, psychological and relational.

The geometry utilized for the head of the Domestic and Condominium robots conforms with the pattern used for the DustCart, the third robot of the fleet. Furthermore, research was carried out for each machine to give the Robot-Era fleet a common identity, that would translate in the greatest recognisability and friendliness. The elements characterising the exterior aspect of each robot are the large dimension of their eyes, to enhance perception and interaction; the personalized headgear, to stress their different functions in a household and external environments; the proportions between the cover components for a precise harmony of colours and materials, to facilitate the product acceptance [16].

The Domestic and Condominium robot head covers stem from the archetypical concept of "clothing-uniform". It means that each robot resembles a specific assistant: a housemaid inside the household and a janitor for public spaces. This last characteristic is conveyed through different colours and different hats for each robot.

The Domestic robot is equipped with a cover that aesthetically resembles a housemaid, while the geometry of the Condominium robot cover uses an actual hat, which typically characterizes a janitor uniform. It is important to add that in both covers teletransparency and recognition systems have been installed, such as cameras in the robot eye zone and a system to track people and environments thanks to optical tools in the area above the eyes (two video cameras and a supplementary infrared detector) for visual recognition of bodies in motion [14].

\subsection{Robot-Robot Interaction}

In the interaction between robots exchanging objects is of pivotal importance. However, in this study several other aspects are also taken into account: from object manipulation performed by the robotic arm (a part of the domestic robot), to the mechanical alignment of the robots when they exchange objects.

The torso of the robots is comprised of a shell with a tablet on its anterior part and this works as a communicative interface between user and robot. In the posterior part of the robot a foldaway support system has been installed so to assist elderly users with reduced mobility in those particularly difficult actions, like getting up from a chair or from the bed.

Objects may be easily transported thanks to a horizontal loading platform positioned in the anterior part of the robot. This device works through motorized rollers that allow an optimal sliding movement. It is worth mentioning that the geometry of the anterior loading platform is designed so that two robots are mechanically aligned. 
This is important because it means that objects may be safely transferred from one platform to the other, with the aid of the mechanical arm of the Domestic robot.
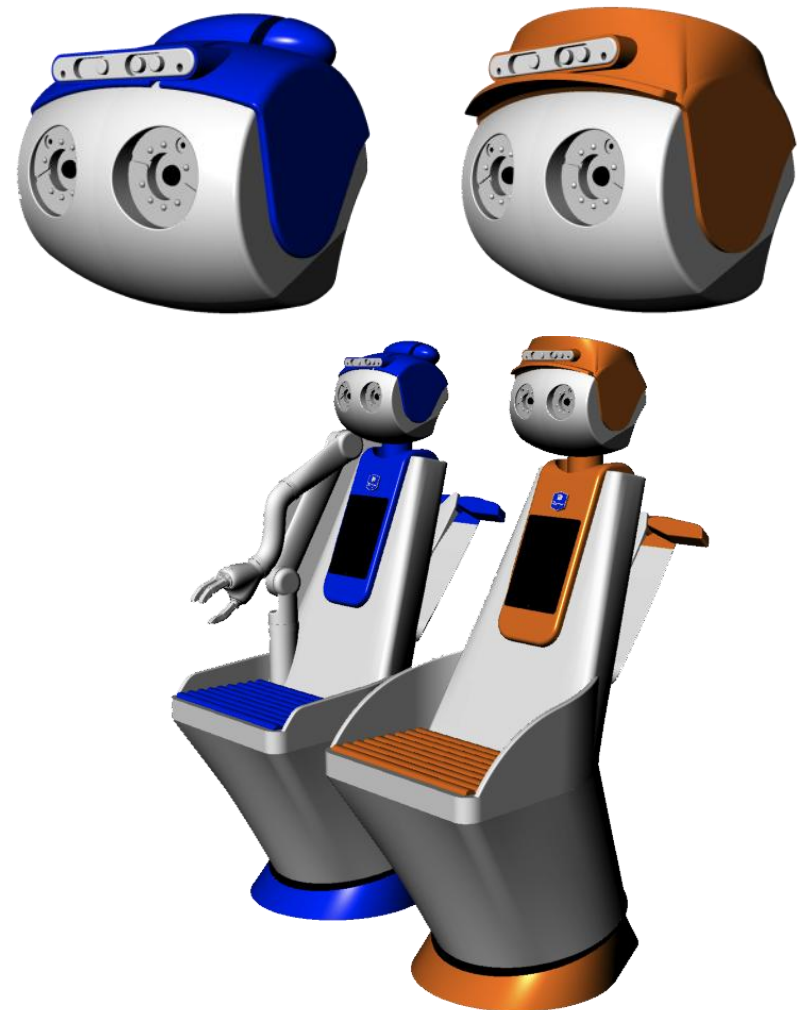

Figure 2 - Concept sketches of the Robot-Era robots: in blue the Domestic Robot with the manipulator and in orange the Condominium robot.

\subsection{Robot Environment Interactions}

Sensors placed in the environment together with sensors in the robot allow machines to move accurately around the given space and this is important because it ensures greater safety for the user.

These robots are programmed to communicate both with their users and the environment where they perform their functions. For this reason they are denominated 
"intelligent machines". They can recognise obstacles inside the household, such as architectural features, furniture and other static objects. Additionally, they are also able to recognise moving objects, thus enabling them to better interact with elderly users, both in their daily routines or in situations of need or danger.

\section{Acknowledge}

The research leading to these results has received funding from the European Community's Seventh Framework Programme (FP7/2007-2013) under grant agreement num. 288899 (Robot-Era Project)

\section{References}

1. “The Global Aging Experience Project. Ethnographic Research”, Intel research, 2010.

2. G. van der Broek, F. Cavallo, and C. Wehrmann, "AALIANCE Ambient Assisted Living Roadmap", Vol. 6, IOS Press, 2010.

3. Eurostat, "European social statistics - edition 2013", 2013.

4. Paolo Dario, Paul F.M.J. Verschure, Tony Prescott, "Robot Compan-ions for Citizens", Procedia Computer Science 7 (2011) 4751.

5. F. Cavallo, M. Aquilano, M. Bonaccorsi, R. Limosani, A. Manzi, M.C. Carrozza, P. Dario, "On the design, development and experimentation of the ASTRO assistive robot integrated in smart environments", IEEE International Conference on Robotics and Automation (ICRA 2013), Karlsruhe, Germany, May 6-10, 2013.

6. Bevilacqua R., Felici E., Marcellini F., Klemcke S., Nedopil C., Glende S., Filippo C., Aquilano M., Carrozza M.C., Dario P., "Robot-Era project: from the end-user's perspective to robotics. Preliminary findings", Ambient Assisted Living Forum, Eindhoven, 2012.

7. Cowan D, Turner-Smith A., "The role of assistive technology in alternative models of care for older people", With respect to old age, Vol.2, Centre of Rehab. Engineering, King's College London, 1999.

8. F. Cavallo, M. Aquilano, M. Bonaccorsi, I. Mannari, M.C. Carrozza, P. Dario, "Multidisciplinary approach for developing a new robotic system for domiciliary assistance to elderly people", 33rd Annual International Conference of the IEEE EMBS Boston, Massachusetts USA, August 30 - September 3, 2011.

9. N. Casiddu, E. Micheli (2011). Human Centered Robotic Design. p. 1-176, Firenze: Alinea Editrice, 2011. 
10. Lazakidou A., Siassiakos K., Konstantinos I., Wireless Technologies for Ambient Assisted Living and Healthcare - Systems and Applications, IGI Global, Hersey, 2010.

11. Albert W., Tedesco D., Tullis T., Beyond the Usability Lab: Conducting Large-scale Online User Experience Studies, Morgan Kaufmann, Boston, 2009.

12. Lupacchini A., Design olistico. Progettare secondo i principi del DfA, Firenze: Alinea, 2010.

13. Del Curto B., Fiorani E., Passaro C., La pelle del design. Progettare la sensorialità, Milano: Lupetti, 2010.

14. Accolla A., Design for All. Il progetto per l'individuo reale, Milano: FrancoAngeli, 2009.

15. Bennett K. B., Flach J. M. - Display and Interface Design: subtle science, exact art, London : CRC Press, 2011.

16. Karwowski W., Rahimi M., Human-Robot Interaction, London: Taylor \& Francis, 2003. 